\title{
MRI-Based Attenuation Correction for Hybrid PET/MRI Systems: A 4-Class Tissue Segmentation Technique Using a Combined Ultrashort-Echo-Time/Dixon MRI Sequence
}

\author{
Yannick Berker ${ }^{1,2}$, Jochen Franke ${ }^{2}$, André Salomon ${ }^{2,3}$, Moritz Palmowski ${ }^{1,4}$, Henk C.W. Donker ${ }^{5}$, Yavuz Temur ${ }^{4}$, \\ Felix M. Mottaghy ${ }^{4,6}$, Christiane Kuhl ${ }^{5}$, David Izquierdo-Garcia ${ }^{7}$, Zahi A. Fayad ${ }^{7}$, Fabian Kiessling ${ }^{1}$, \\ and Volkmar Schulz ${ }^{1,2}$ \\ ${ }^{I}$ Department of Experimental Molecular Imaging, RWTH Aachen University Hospital, Aachen, Germany; ${ }^{2}$ Philips Technologie \\ GmbH Innovative Technologies, Research Laboratories, Aachen, Germany; ${ }^{3}$ Division of Imaging Sciences and Biomedical \\ Engineering, King's College London, St. Thomas Hospital, London, United Kingdom; ${ }^{4}$ Department of Nuclear Medicine, RWTH \\ Aachen University Hospital, Aachen, Germany; ${ }^{5}$ Department of Diagnostic and Interventional Radiology, RWTH Aachen University \\ Hospital, Aachen, Germany; ${ }^{6}$ Department of Nuclear Medicine, Maastricht University Medical Center, Maastricht, The Netherlands; \\ and ${ }^{7}$ Imaging Science Laboratories, Translational and Molecular Imaging Institute, Department of Radiology, Mount Sinai School of \\ Medicine, New York, New York
}

Accurate $\gamma$-photon attenuation correction (AC) is essential for quantitative $\mathrm{PET} / \mathrm{MRI}$ as there is no simple relation between MR image intensity and attenuation coefficients. Attenuation maps ( $\mu$-maps) can be derived by segmenting MR images and assigning attenuation coefficients to the compartments. Ultrashortecho-time (UTE) sequences have been used to separate cortical bone and air, and the Dixon technique has enabled differentiation between soft and adipose tissues. Unfortunately, sequential application of these sequences is time-consuming and complicates image registration. Methods: A UTE triple-echo (UTILE) MRI sequence is proposed, combining UTE sampling for bone detection and gradient echoes for Dixon water-fat separation in a radial 3-dimensional acquisition (repetition time, $4.1 \mathrm{~ms}$; echo times, 0.09/1.09/2.09 ms; field strength, $3 \mathrm{~T}$ ). Air masks are derived mainly from the phase information of the first echo; cortical bone is segmented using a dual-echo technique. Soft-tissue and adipose-tissue decomposition is achieved using a 3-point Dixon-like decomposition. Predefined linear attenuation coefficients are assigned to classified voxels to generate MRI-based $\mu$-maps. The results of 6 patients are obtained by comparing $\mu$-maps, reciprocal sensitivity maps, reconstructed PET images, and brain region PET activities based on either CT AC, two 3-class MRI AC techniques, or the proposed 4-class UTILE AC. Results: Using the UTILE MRI sequence, an acquisition time of $214 \mathrm{~s}$ was achieved for the head-and-neck region with 1.75-mm isotropic resolution, compared with $164 \mathrm{~s}$ for a single-echo UTE scan. MRI-based reciprocal sensitivity maps show a high correlation with those derived from CT scans $\left(R^{2}=\right.$ 0.9920). The same is true for PET activities $\left(R^{2}=0.9958\right)$. An overall voxel classification accuracy (compared with $C T$ ) of $81.1 \%$ was reached. Bone segmentation is inaccurate in complex regions such as the paranasal sinuses, but brain region

\footnotetext{
Received May 19, 2011; revision accepted Dec. 16, 2011.

For correspondence or reprints contact: Yannick Berker, Department of Experimental Molecular Imaging, RWTH Aachen University Hospital, Pauwelsstrasse 30, 52074 Aachen, Germany.

E-mail: yberker@ukaachen.de

Published online Apr. 13, 2012.

COPYRIGHT $\odot 2012$ by the Society of Nuclear Medicine, Inc.
}

activities in 48 regions across 6 patients show a high correlation after MRI-based and CT-based correction $\left(R^{2}=0.9956\right)$, with a regression line slope of 0.960 . All overall correlations are higher and brain region PET activities more accurate in terms of mean and maximum deviations for the 4-class technique than for 3-class techniques. Conclusion: The UTILE MRI sequence enables the generation of MRI-based 4-class $\mu$-maps without anatomic priors, yielding results more similar to CT-based results than can be obtained with 3-class segmentation only.

Key Words: PET; MRI; molecular imaging; attenuation correction; ultrashort echo time

J Nucl Med 2012; 53:796-804

DOI: 10.2967/jnumed.111.092577

$\mathbf{H}$ ybrid medical imaging systems-for example, comprising both a PET and a CT imaging device-have evolved into standard diagnostic tools in clinical routine within the last decade (1). Recently, a hybrid system combining a PET device and an MRI system with simultaneous PET/MRI data acquisition has been presented $(2,3)$, and clinical systems are already being marketed. Compared with CT, MRI provides versatile soft-tissue contrast, yielding superior diagnostic accuracy without subjecting the patient to ionizing radiation. Furthermore, MRI not only reveals the essential morphologic information but also provides sophisticated pulse sequences such as diffusion-weighted imaging or MR spectroscopy (4) to visualize tissue function. Finally, the combination of PET and MRI has potential distinct advantages over PET/CT such as the ability to perform motion correction of PET data.

However, practical PET/MRI implementations still suffer from the lack of accurate MRI-based methods for attenuation correction (AC) of the measured PET emission data. 
Yet, correcting for $\gamma$-photon attenuation caused by the patient, the examination table, and the MRI coils is an imperative requirement of quantitative PET data analysis. Although CT-based AC in PET/CT systems can be considered straightforward, as $\mathrm{CT}$ measures the attenuation coefficient of tissues at $\mathrm{X}$-ray energies, MRI-based AC is challenging. Because the MRI signal is determined by tissue hydrogen density and relaxation properties, the derivation of 511-keV-photon attenuation coefficients is much more complex than in CT AC.

Different atlas- or classification-based MRI AC techniques have recently been reported (5-10). Current atlas-based approaches cannot entirely cope with interpatient variations and abnormalities such as bone resections or implants; therefore, the focus of this study is on approaches based on automatic tissue classification of MRI data. Concerning the choice of tissue classes, apart from air and soft tissue, Schulz et al. (7) have shown that adipose tissue has to be considered in whole-body imaging if accurate $\mathrm{AC}$ is desired. The same has been stated by Martinez-Möller et al. ( 8 ), who have proposed a 4-tissue-class segmentation (air, lung, soft tissue, and adipose tissue) using a Dixon method for whole-body MRI. In head datasets, cortical bone has been found to be an important tissue class for accurate AC by Catana et al. (9). Unfortunately, bone is difficult to distinguish from air in conventional MRI sequences because of extremely short $T_{2}$ relaxation times, resulting in a fast-decaying bone signal (11). Therefore, Keereman et al. have proposed the use of ultrashort-echo-time (UTE) sequences for cortical bone segmentation in the head (10).

The combination of cortical bone segmentation and water-fat separation is a promising approach to integrating all tissue classes relevant for attenuation in head-and-neck PET/MRI. However, the total acquisition time should be used primarily for diagnostic scans, and sequential application of UTE and Dixon MRI sequences constitutes a suboptimal solution. Therefore, we propose a combined MRI sequence for the discrimination of cortical bone, air, adipose tissue, and soft tissue in head-and-neck PET/MRI. For evaluation, we compare the resulting 4-class MRI AC technique with 3-class MRI AC approaches and with CT AC.

\section{MATERIALS AND METHODS}

\section{Volunteer and Patient Studies}

Experiments on 1 healthy volunteer and 6 patients were performed as approved by the institutional ethics committee. Volunteer and patients had no contraindication to an MRI examination, and they gave written consent to be investigated using the proposed MRI sequence.

All patients included in this study had undergone a head-andneck ${ }^{18}$ F-FDG PET/CT examination (Gemini TF 16 PET/CT scanner; Philips) for cancer staging.

\section{MRI Scanner}

All measurements were performed on a clinical Achieva 3.0-T TX system using a SENSE NeuroVascular 16-channel receiver coil (Philips) with CLEAR (Constant LEvel AppeaRance). The minimum achievable echo time (TE) for UTE acquisition is ruled by the transmitter and the receiver coils, as the energy stored in the transmit coil must ring down before a safe tuning of the receiver coil can start. Although ringing down typically takes only a few microseconds (12), tuning of the coils can be more time-consuming. A tune-delay parameter of $50 \mu \mathrm{s}$ was selected via a coil configuration software patch, resulting in a minimum first echo time of $\mathrm{TE}_{1}=90 \mu \mathrm{s}$.

\section{MRI Pulse Sequence}

The proposed UTE triple-echo (UTILE) sequence (Fig. 1) combines UTE acquisition for signal reception from fast-decaying tissue types and water-fat signal decomposition following the Dixon scheme. It features a nonselective radiofrequency blockpulse for volumetric excitation (flip angle: $10^{\circ}$, pulse width: 51 $\mu s)$; the sampling of the UTE signal starts at the same time as the ramp of the readout gradient. Starting from the center, k-space is traversed radially outward, with subsequent readouts covering a sphere in k-space. The acquisition sampling-window duration $\mathrm{T}_{\mathrm{AQ} 1}$ is $0.31 \mathrm{~ms}$ per k-space line. Signal decay during acquisition is not corrected for, so that $\mathrm{T}_{\mathrm{AQ} 1}$ directly affects resolution and signal level of short- $\mathrm{T}_{2}$ components in the image (12).

Echo times of gradient echoes are such that spins associated with water and fat are almost opposite each other $\left(\mathrm{TE}_{2}=1.09\right.$ $\mathrm{ms})$ and in-phase $\left(\mathrm{TE}_{3}=2.09 \mathrm{~ms}\right)$, respectively; acquisition window durations are $\mathrm{T}_{\mathrm{AQ} 2}=\mathrm{T}_{\mathrm{AQ} 3}=0.49 \mathrm{~ms}$. Spins are dephased using a crusher gradient; the repetition time (TR) is $4.1 \mathrm{~ms}$. The field of view of $280^{3} \mathrm{~mm}^{3}$ covers the complete head-and-neck area (matrix size [acquisition/reconstruction]:
FIGURE 1. Timing diagram of UTILE MRI sequence; $\mathrm{RF}$ = radiofrequency excitation; $\mathrm{G}=$ readout gradient; $\mathrm{AQ}=$ data acquisition.

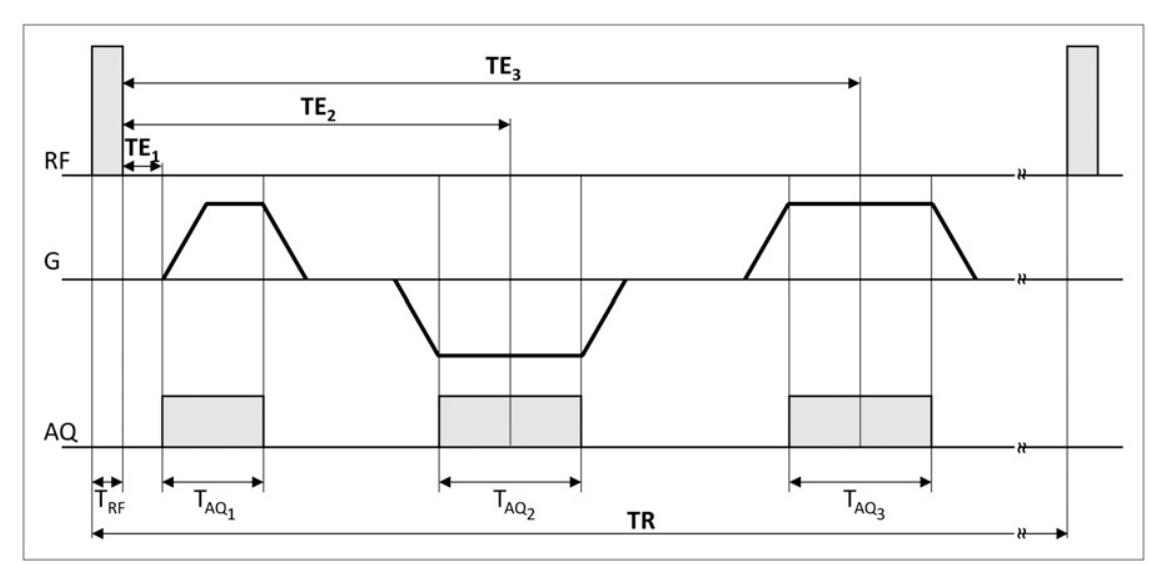


$160^{3} / 240^{3}$; voxel size [acquisition/reconstruction]: $1.75^{3} / 1.17^{3}$ $\mathrm{mm}^{3}$ ); the total acquisition time is $214 \mathrm{~s}$. Reconstructed complex images are denoted $I_{n}=M_{n} \cdot e^{j \phi_{n}}, n \in\{1,2,3\}$ using their magnitude $M_{n}$ and phase $\phi_{n}$; any location dependence is omitted for ease of notation.

To allow for a quantitative assessment of the proposed water-fat decomposition, an additional 2-dimensional mDixon (Philips) acquisition as integrated in the MRI scanner software was performed for the volunteer $\left(\mathrm{TR} / \mathrm{TE}_{1} / \mathrm{TE}_{2} / \mathrm{TE}_{3}=11 / 4.6 / 5.1 / 5.6 \mathrm{~ms}\right.$; field of view: $280^{2} \mathrm{~mm}^{2}$; flip angle: $15^{\circ}$; prepulse: inversion; inversion time: $800 \mathrm{~ms}$; matrix size [acquisition/reconstruction]: $187^{2} / 320^{2}$; pixel size [acquisition/reconstruction]: $1.5^{2} / 0.875^{2} \mathrm{~mm}^{2}$; slice thickness: $4 \mathrm{~mm}$; reconstructed images: water and fat).

\section{MRI Postprocessing}

Postprocessing of reconstructed MRI data was performed in MATLAB (R2011a; The MathWorks). The effect of different coil sensitivities in the gradient echoes is accounted for in image reconstruction using a SENSE reference scan; inhomogeneous sensitivities in the UTE echo were compensated for by exploiting the similarity of heavily smoothed $\mathrm{M}_{1}$ and $\mathrm{M}_{3}$, both acquired approximately under in-phase conditions. An air mask, including cavities, was then created using magnitude and phase thresholding of mainly the first echo as well as morphologic filtering and connected-component analysis.

Cortical bone was segmented using a dual-echo technique (13) to detect the MRI signal originating from short- $\mathrm{T}_{2}$ components. Therefore, the relative signal decay between $M_{1}$ and $M_{3}$ was computed by

$$
d_{U T E}=2 \cdot \frac{M_{1}-M_{3}}{M_{1}+M_{3}}
$$

$\left(\mathrm{d}_{\mathrm{UTE}}=\right.$ differential UTE signal $)$ and masked by the air mask to yield $\mathrm{d}_{\mathrm{UTE}}^{\mathrm{m}}$ (masked $\mathrm{d}_{\mathrm{UTE}}$; see flow chart in Supplemental Fig. 1 [supplemental materials are available online only at http://jnm. snmjournals.org]). Bony structures were presegmented using an empirically determined global threshold $(\mathrm{t}=0.55)$ and cleaned up using several steps of morphologic filtering and connectedcomponent analysis.

The remaining tissue (everything except air and bone) is assigned a relative water-fat fraction, determined through an adapted Dixon decomposition $(14,15)$. For this UTILE-based 3-point Dixon method, signals are described by their magnitude and unwrapped phase; the latter is recovered using a 3-dimensional phase-unwrapping algorithm (16). Each signal intensity $I_{n}$ is assumed to be composed of 2 signal fractions, described by their magnitudes $M_{w}$ (water) and $M_{f}$ (fat), respectively. Their relative phases are determined by the unknown magnetic field inhomogeneities $\Delta B_{0}$ and the chemical shift of fat of $\Delta \omega_{f} / \omega=3.5 \mathrm{ppm}$ (17). With the proton's gyromagnetic ratio $\gamma$ and an unknown static phase offset $\varphi^{s}$, the signal model of echo $n$ reads

$$
I_{n}=\left[M_{w}+M_{f} \cdot e^{j \cdot \Delta \omega_{f} \cdot T E_{n}}\right] \cdot e^{j \cdot\left(\phi^{s}+\gamma \cdot \Delta B_{0} \cdot T E_{n}\right)} .
$$

Using the approximations $T E_{1} \approx 0$ and $e^{j \cdot \Delta \omega_{f} \cdot T E_{3}} \approx 1$, one derives $\phi^{s}$ and $\Delta B_{0}$ from $I_{1}$ and $I_{3} / I_{1}$. This allows solving the linear equation system $\left\{I_{2}, I_{3}\right\}$ for $M_{w}$ and $M_{f}$. From these, one calculates the water-fat fraction $r=\left(M_{w}-M_{f}\right) /\left(M_{w}-M_{f}\right)$, ranging continuously from -1 (purely adipose tissue) to +1 (purely soft tissue), which is physiologically more meaningful than a binary classification.
TABLE 1

Linear Attenuation Coefficients and Windows for Generation and Segmentation of $\mu$-Maps*

\begin{tabular}{lccc}
\hline & & \multicolumn{2}{c}{ Segmentation } \\
\cline { 3 - 4 } Segment & Generation & Lower & Upper \\
\hline Air & 0 & 0 & $0.040 \mathrm{~cm}^{-1}$ \\
Adipose & $0.090 \mathrm{~cm}^{-1}(21)$ & $0.040 \mathrm{~cm}^{-1}$ & $0.095 \mathrm{~cm}^{-1}$ \\
Soft & $0.100 \mathrm{~cm}^{-1}(9)$ & $0.095 \mathrm{~cm}^{-1}$ & $0.110 \mathrm{~cm}^{-1}$ \\
Bone & $0.172 \mathrm{~cm}^{-1}(22)$ & $0.110 \mathrm{~cm}^{-1}$ & $\infty$ \\
& & & \\
& & & \\
\hline & &
\end{tabular}

\section{Attenuation Map ( $\mu$-Map) Generation}

Tissue-specific linear attenuation coefficients for $511-\mathrm{keV}$ photons were extracted from the literature (Table 1). While population of the segmented image is straightforward for air and bone segments, the mean attenuation coefficient for each mixed waterfat voxel is calculated from the relative water-fat fraction $r$ using the linear mapping

$$
\mu_{r}=\frac{1+r}{2} \cdot \mu_{\text {soft }}+\frac{1-r}{2} \cdot \mu_{\text {adipose }} .
$$

A low-dose CT (matrix size: $512 \times 512$; voxel size: $1.17 \times 1.17 \times$ $5 \mathrm{~mm}$; effective tube-current-time product: $30 \mathrm{mAs}$; tube voltage: $120 \mathrm{kVp}$ ) was rescaled (7) to provide reference $\mu$-maps denoted $\mu_{\mathrm{CT}}$. Patient $\mu$-maps derived from MRI were manually coregistered to $\mu_{\mathrm{CT}}$ by rigid transformation and resampled to CT resolution using commercially available software (IMALYTICS Research Workstation Prerelease; Philips Research). To focus on the evaluation of tissue classification performance, $\mu_{\mathrm{CT}}$ was copied to yield an initial $\mu_{\text {UTILE }}$. Then, the patient's body was segmented and deleted from $\mu_{\text {UTILE }}$ by thresholding and morphologic filtering, and the respective voxels were copied from the coregistered MRI-based $\mu$-map.

For each 4-class $\mu$-map, two 3-class $\mu$-maps were created: skipping the water-fat separation, one obtains $\mu_{\text {MRI }}^{\text {NoDixon }}$ with $\mu_{\mathrm{MRI}}^{\text {NoDixon }}=\mu_{\mathrm{soft}}$ in water-fat voxels; skipping bone segmentation, one obtains $\mu_{\mathrm{MRI}}^{\mathrm{NoUTE}}$ with $\mu_{\mathrm{MRI}}^{\mathrm{NoUTE}}=\mu_{\mathrm{r}}$ in bone voxels. Workflow and registration parameters were identical to the generation of $\mu_{\text {UTILE. }}$
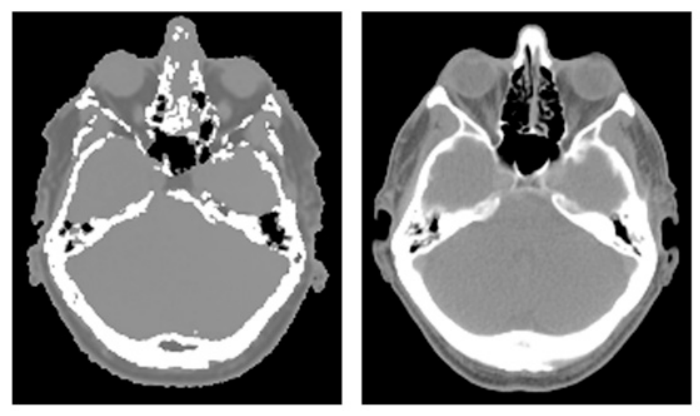

FIGURE 2. Corresponding transverse slices of $\mu_{U T I L E}$ (left) and $\mu_{C T}$ (right) of patient P2 in $\mathrm{cm}^{-1}$. Supplemental Figure 3 provides additional examples. 
TABLE 2

Coclassification of Voxels in $\mu_{C T}$ and $\mu_{U T I L E}$ : Number of Voxels in Intersection of Segmented Tissue Classes Aggregated over All Patients*

\begin{tabular}{|c|c|c|c|c|c|}
\hline CTNUTILE & Air & Adipose & Soft & Bone & Total \\
\hline Air & $5,139,253$ & 79,481 & 76,695 & 10,819 & $5,306,248$ \\
\hline Adipose & 318,798 & 347,219 & 283,857 & 26,944 & 976,818 \\
\hline Soft & 350,787 & 241,274 & $2,124,498$ & 80,966 & $2,797,525$ \\
\hline Bone & 32,146 & 116,671 & 217,109 & 285,943 & 651,869 \\
\hline Total & $5,840,984$ & 784,645 & $2,702,159$ & 404,672 & $9,732,460$ \\
\hline
\end{tabular}

\section{Evaluation of $\mu$-Maps}

$\mu_{\mathrm{CT}}$ and $\mu_{\mathrm{UTILE}}$ voxels were classified into 4 tissue classes based on voxel intensities (Table 1). The classification was restricted to a minimum rectangular bounding box of voxels classified as "body" in the MRI data of each patient. Linear attenuation coefficient windows for classification were chosen to take partialvolume effects (CT) and interpolation effects at tissue interfaces (MRI) into account. To quantify misclassifications, the number of voxels in the intersection of tissue classes in $\mu_{\mathrm{CT}}$ and $\mu_{\mathrm{UTILE}}$ was determined. Based on the CT classification, $\mu_{\mathrm{UTILE}}$ and $\mu_{\mathrm{CT}}$ values in each tissue class were compared.

All $\mu_{\mathrm{x}}$ were slicewise Radon-transformed (18) to yield the respective sinograms $\sigma_{\mathrm{x}}$, representing the integrated attenuation coefficient along all in-slice lines of response. Following the BeerLambert law, the attenuation along a line of response is $\exp \left(-\sigma_{x}\right)$. The inverse Radon transform of the attenuation was calculated without filtering to yield a sensitivity map (this step, an unfiltered backprojection, is equivalent to averaging all sinogram entries containing a contribution from a voxel in image space, and yields a result in image space). In the scope of this work, the reciprocal value of a sensitivity map is called a sensitivity correction map $s_{\mathrm{x}}$, quantifying in-slice attenuation effects. $s_{x}$ is supposed to be an estimate of the AC influence on the reconstructed PET radiation intensities, considering the used reconstruction code (19). Linear correlation of $\mathrm{s}_{\mathrm{UTILE}}$ and both $\mathrm{s}_{\mathrm{MRI}}$ and $\mathrm{s}_{\mathrm{CT}}$ was evaluated on a perpatient basis, as well as aggregated over all patients.

\section{Evaluation Using PET Data}

Measured PET emission data were corrected for random and scattered coincidences and attenuation on the basis of the respective $\mu$-maps during list-mode ordered-subset expectation maximization reconstruction (19) (9 iterations; number of subsets used in iterations 19: $16,8,4,4,2,2,1,1,1 ; 288 \times 288 \times 130$ voxels; 2 bed positions; voxel size: $2.0375^{3} \mathrm{~mm}^{3}$ ). Because of undesired movement of 1 patient during PET acquisition, the reconstruction was limited to 1 bed position $(288 \times 288 \times 88$ voxels $)$ in this patient. PET image data were scaled to standardized uptake values (SUVs): after extracting a proportionality factor between the $\mathrm{PET}_{\mathrm{CT}}$ and the PET reconstruction obtained from the PET scanner software for each patient, the same factor was applied to all PET reconstructions $\left(\mathrm{PET}_{\mathrm{CT}}, \mathrm{PET}_{\mathrm{UTILE}}\right.$, and $\mathrm{PET}_{\mathrm{MRI}}^{\mathrm{NoDixon} / \mathrm{NoUTE}}$ ) of a given patient.

The reconstructed PET data were smoothed using a 3-dimensional gaussian low-pass filter (full width at half maximum, 3 voxels), and linear correlation of $\mathrm{PET}_{\mathrm{UTILE}}$, $\mathrm{PET}_{\mathrm{MRI}}^{\mathrm{NoDixon}}$, and $\mathrm{PET}_{\mathrm{MRI}}^{\mathrm{NoUTE}}$ with $\mathrm{PET}_{\mathrm{CT}}$ was evaluated. In addition, the relative difference between PET $_{\mathrm{CT}}$ and all $\mathrm{PET}_{\mathrm{UTILE} / \mathrm{MRI}}$ datasets was calculated voxelwise following $\delta_{x}=\left(P E T_{x}-P E T_{C T}\right) / P E T_{C T}$. These analyses were re- stricted to slices that were part of both MRI and CT field of views. Slices in which metallic tooth implants caused CT artifacts were excluded in the case of 4 patients.

Finally, to evaluate the impact on brain PET examinations, 8 regions of interest of volumes between 2 and $9 \mathrm{~mL}$ were manually placed in the brain region of all patients, and the correlation of mean values and SD in these regions of interest was evaluated.

\section{Statistical Analysis}

The significance of correlation coefficients (Pearson) was assessed using the Student $t$ test $\left(t=R \sqrt{\frac{n-2}{1-R^{2}}}, \mathrm{n}-2 \mathrm{df}\right)$; for differences between 2 correlation coefficients, a $z$ test with $z=\left(Z_{f, 1}-Z_{f, 2}\right) \cdot \sqrt{\frac{N-3}{2}}$, the Fischer transform $Z_{f, i}=\frac{1}{2} \cdot \ln \left(\frac{1+R_{i}}{1-R_{i}}\right)$, and the number of voxels $N=N_{1}=N_{2}$ was used. Using the $\mu_{\mathrm{CT}}$ segmentation as a reference, $\mu_{\text {UTILE }}$ values in all tissue classes were compared with those in all other tissue classes using a Wilcoxon rank-sum test. With Wilcoxon signed-rank sums, the relations between different correlation coefficients and the relations between the deviations of regression lines from the $45^{\circ}$ line $(|m-1|$ with slope $\mathrm{m})$ were tested for significance.

\section{RESULTS}

Only 1 patient reported peripheral nerve stimulation, which was not experienced as uncomfortable.

\section{$\mu$-Maps}

Focusing on water-fat separation, the proposed UTILEbased 3-point Dixon reconstruction yields a water-fat fraction $r$ similar to the one based on an established mDixon acquisition and decomposition (Supplemental Fig. 2). The difference between both $r$ in the head voxels shown is $-0.029 \pm$ 0.186 (mean $\pm \mathrm{SD}$ ).

\section{TABLE 3}

Voxel Numbers and $\mu_{C T}$ and $\mu_{U T I L E}$ Intensities (Mean \pm SD) per Region Defined by CT Segmentation (Aggregated over All Patients)

\begin{tabular}{lrrr}
\hline \multicolumn{1}{c}{ Region } & \multicolumn{1}{c}{$n$} & $\mu_{\mathrm{CT}} / \mathrm{Cm}^{-1}$ & $\mu_{\mathrm{UTILE}} / \mathrm{cm}^{-1}$ \\
\hline $\mathrm{ROI}_{\mathrm{CT}, \text { air }}$ & $5,627,857$ & $0.003 \pm 0.006$ & $0.003 \pm 0.016$ \\
$\mathrm{ROI}_{\mathrm{CT} \text {,adipose }}$ & $1,095,530$ & $0.083 \pm 0.014$ & $0.064 \pm 0.045$ \\
$\mathrm{ROI}_{\mathrm{CT}, \text { soft }}$ & $2,973,290$ & $0.099 \pm 0.003$ & $0.086 \pm 0.034$ \\
$\mathrm{ROI}_{\mathrm{CT} \text {,bone }}$ & 714,109 & $0.134 \pm 0.017$ & $0.116 \pm 0.040$ \\
& & & \\
\multicolumn{2}{l}{$\mathrm{ROI}=$ region of interest. } & & \\
\hline
\end{tabular}


TABLE 4

Coefficients of Determination $\left(R^{2}\right)$ Between CT-Based and MRI-Based Sensitivity Correction Maps*

\begin{tabular}{llll}
\hline \multicolumn{1}{c}{ ID } & SUTILE & $\mathrm{s}_{\mathrm{MRI}}^{\text {NoDixon }}$ & $\mathrm{S}_{\mathrm{MRI}}^{\text {NoUTE }}$ \\
\hline P1 & 0.9904 & 0.9895 & 0.9888 \\
P2 & 0.9957 & 0.9952 & 0.9932 \\
P3 & 0.9925 & 0.9914 & 0.9927 \\
P4 & 0.9943 & 0.9935 & 0.9909 \\
P5 & 0.9909 & 0.9903 & 0.9922 \\
P6 & 0.9960 & 0.9953 & 0.9931 \\
Aggregated & 0.9920 & 0.9912 & 0.9897 \\
\hline
\end{tabular}

*All correlations and differences between any $R^{2}$ in SUTILE and corresponding value in any $\mathrm{S}_{\mathrm{MRI}}$ are statistically significant $(P<$ $\left.10^{-6}\right)$.

The 4-class $\mu_{\text {UTILE }}$ exhibits a high visual similarity to $\mu_{\mathrm{CT}}$ despite challenging anatomic regions as in the paranasal sinuses (Fig. 2; Supplemental Fig. 3). The fraction of correctly classified voxels was over $80 \%$ for all patients, with an aggregated $81.1 \%$ (Table 2). Mean $\mu_{\text {UTILE }}$ intensities are found to be pairwise significantly different across tissue classes (all $P<10^{-6}$ ), signifying that $\mu_{\text {UTILE }}$ in a voxel depends on the tissue class of the corresponding CT voxel (Table 3 ). Differences between mean $\mu_{\mathrm{CT}}$ and $\mu_{\mathrm{UTILE}}$ intensities in the same tissue class are either small (air voxels) or can be explained by partial-volume effects and misregistration, both inducing misclassifications (e.g., between soft-tissue and bone voxels).

\section{Sensitivity Correction Maps}

Correlations between sensitivity correction maps (Table 4) are high and slopes of the linear regression lines (Table 5) close to 1; compare Figure 3 for aggregated and Supplemental Figure 4 for individual patient data. SUTILE correlates better with $s_{\mathrm{CT}}$ than any $\mathrm{s}_{\mathrm{MRI}}$ in aggregated data; in individual patients, $s_{U T I L E}$ correlation is higher than $s_{\text {MRI }}^{\text {NoDixon }}$ correlation in all cases $(P=0.031)$ and better than $\mathrm{s}_{\mathrm{MRI}}^{\mathrm{NoUTE}}$ correlation in 4 of 6 individual patients.

Similarly, the slope of the regression line is closer to 1 for $\mathrm{s}_{\mathrm{UTILE}}(0.997)$ than for any $\mathrm{s}_{\mathrm{MRI}}$ in aggregated data (1.050 for $\mathrm{s}_{\text {MRI }}^{\text {NoDixon }}$ and 0.893 for $\mathrm{s}_{\text {MRI }}^{\text {NoUTE }}$ ). In individual patient data, the slopes for $\mathrm{s}_{\text {UTILE }}$ deviate less from 1

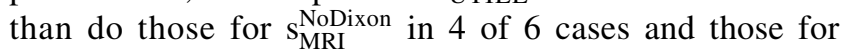
$\mathrm{s}_{\text {MRI }}^{\text {NoUTE }}$ in all cases $(P=0.031)$.

\section{PET Activities}

The visual comparison of reconstructed PET data, using different $\mu$-maps for AC, illustrates the performance (Fig. 4; Supplemental Fig. 5). The joint histograms of PET voxel intensities confirm this observation (all patients aggregated: Fig. 5; individual patients: Supplemental Fig. 6), and the improved correlation of $\mathrm{PET}_{\mathrm{UTILE}}$ with $\mathrm{PET}_{\mathrm{CT}}$, compared with both $\mathrm{PET}_{\mathrm{MRI}}$, is in line with the aforementioned results of sensitivity corrections (Table 6). Correlation coefficients of PET $_{\text {UTILE }}$ are higher than those of PET $_{\text {MRI }}^{\text {NoDixon and }}$ $\mathrm{PET}_{\mathrm{MRI}}^{\mathrm{NoUTE}}$ in all cases except one $\left(\mathrm{PET}_{\mathrm{MRI}}^{\text {NoDixon in patient }}\right.$ $\left.\mathrm{P} 4 ; \Delta\left(R^{2}\right)=1.6 \times 10^{-5}\right)$.

The regression line slopes of sensitivity correction maps translate into regression line slopes in PET activities (Table 7) with high correlation as hypothesized above, although the range of PET activity slopes is narrower than the range of sensitivity correction map slopes (regression line between sensitivity correction slopes and PET activity slopes: $y=0.760 x+0.232 ; R^{2}=0.953$ ).

The average difference between the PET $_{\text {UTILE }}$ and the $\mathrm{PET}_{\mathrm{CT}}$ datasets, aggregated over all patients in all regions where $\mathrm{PET}_{\mathrm{CT}}$ is greater than $0.2 \mathrm{SUV}$, is an overestimation of the activity by $0.001 \mathrm{SUV} \pm 0.058 \mathrm{SUV}$ (mean $\pm \mathrm{SD}$ ); the average absolute difference is $0.010 \mathrm{SUV} \pm 0.057 \mathrm{SUV}$. Considerable differences $\left(\delta_{\min }=-2.3 \mathrm{SUV}, \delta_{\max }=+4.6\right.$ SUV) are observed within regions that are challenging with regard to cortical bone segmentation, such as the labyrinth of ethmoid and the pharynx. For comparison, $\mathrm{PET}_{\mathrm{MRI}}^{\mathrm{NoDix}}$ shows $\delta_{\min }=-2.3 \mathrm{SUV}$ and $\delta_{\max }=+4.9 \mathrm{SUV}$, whereas in $\mathrm{PET}_{\mathrm{MRI}}^{\mathrm{NoUTE}}, \delta_{\min }=-4.1 \mathrm{SUV}$ and $\delta_{\max }=+2.5 \mathrm{SUV}$ are observed. Both $\mathrm{PET}_{\mathrm{MRI}}^{\mathrm{NoDix}}$ and $\mathrm{PET}_{\mathrm{MRI}}^{\mathrm{NoUTE}}$ yield higher mean differences and mean absolute differences than PET $_{\text {UTILE. }}$

In the 48 evaluated brain regions ( 8 regions $\times 6$ patients; compare Supplemental Fig. 7), mean PET $_{\text {UTILE }}$ activities show differences between $-4.8 \%$ and $+7.6 \%$ (mean \pm SD: $0.00 \pm 0.15 \mathrm{SUV})$, compared with up to $+11.4 \%$ in $\operatorname{PET}_{\text {MRI }}^{\text {NoDixon }}(0.18 \pm 0.14 \mathrm{SUV})$ and down to $-14.1 \%$ in $\operatorname{PET}_{\text {MRI }}^{\text {NoUTE }}(-0.30 \pm 0.25$ SUV $)$. Significant correlation is found between the regional mean values $\left(R^{2}=0.9956, P<\right.$ $\left.10^{-6}\right)$ and regional SD $\left(R^{2}=0.9874, P<10^{-6}\right)$ of $\mathrm{PET}_{\mathrm{CT}}$ and PET $_{\text {UTILE }}$ (Fig. 6), both being higher than the corresponding values for $\mathrm{PET}_{\mathrm{MRI}}^{\mathrm{NoDix}}$ an and $\mathrm{PET}_{\mathrm{MRI}}^{\mathrm{NoUTE}}$.

TABLE 5

Regression Lines Between CT-Based and MRI-Based Sensitivity Correction Maps

\begin{tabular}{|c|c|c|c|}
\hline ID & SUTILE & $\mathrm{S}_{\mathrm{MRI}}^{\text {NoDixon }}$ & $\mathrm{S}_{\mathrm{MRI}}^{\text {NoUTE }}$ \\
\hline $\mathrm{P} 1$ & $1.033 x-0.026$ & $1.088 x-0.075$ & $0.944 x+0.054$ \\
\hline P2 & $0.975 x+0.019$ & $1.028 x-0.028$ & $0.861 x+0.122$ \\
\hline P3 & $1.024 x-0.023$ & $1.082 x-0.078$ & $0.904 x+0.090$ \\
\hline P4 & $0.953 x+0.040$ & $1.002 x-0.002$ & $0.852 x+0.127$ \\
\hline P5 & $1.004 x-0.005$ & $1.050 x-0.045$ & $0.915 x+0.073$ \\
\hline P6 & $0.969 x+0.025$ & $1.020 x-0.020$ & $0.854 x+0.126$ \\
\hline Aggregated & $0.997 x+0.001$ & $1.050 x-0.045$ & $0.893 x+0.095$ \\
\hline
\end{tabular}




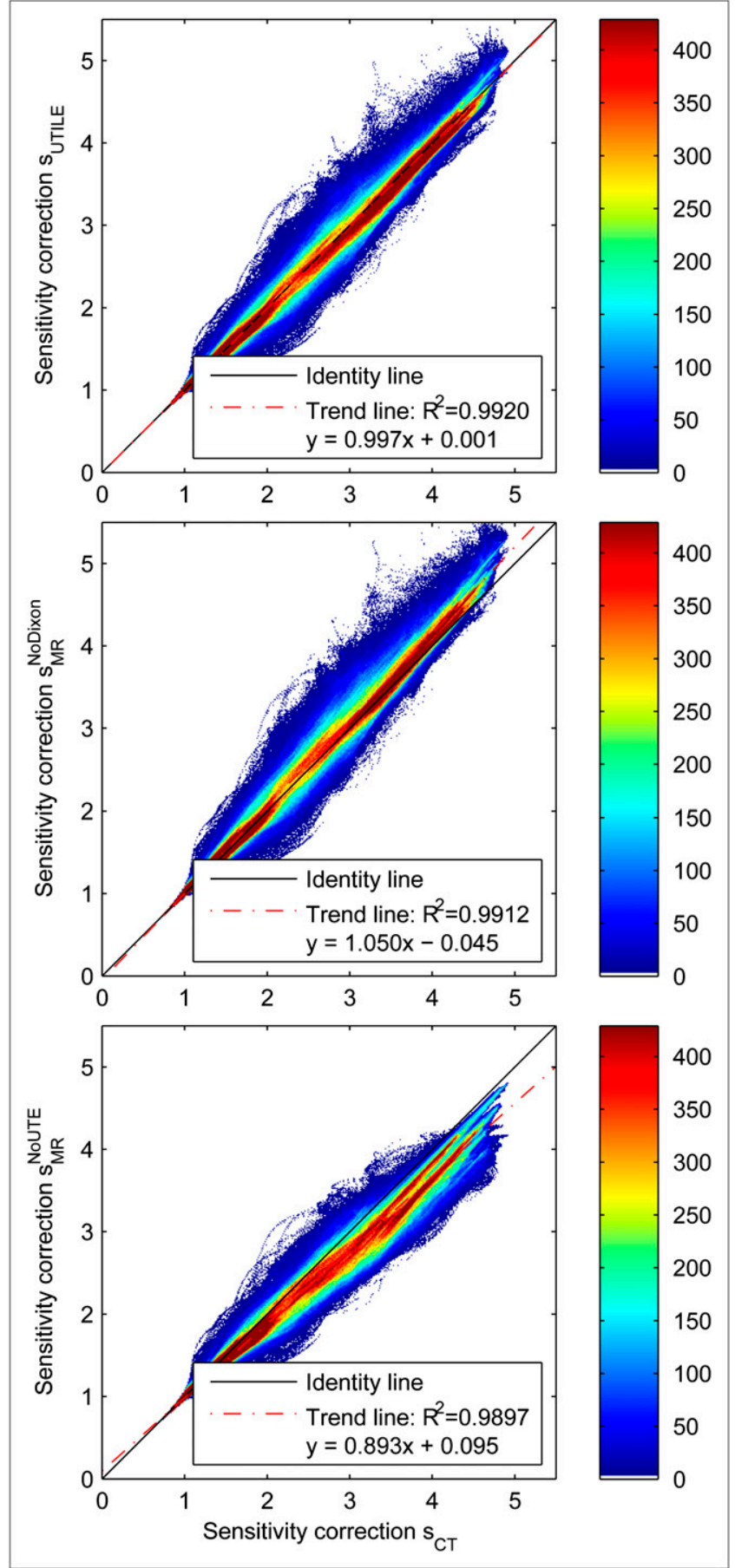

FIGURE 3. Joint histograms of sensitivity correction maps SUTLEE (top), $s_{\mathrm{MRI}}^{\text {NoDixon }}$ (middle), and $\mathrm{s}_{\mathrm{MRI}}^{\text {NoUTE }}$ (bottom), all correlated with $\mathrm{s}_{\mathrm{CT}}$ (aggregated over all patients). Supplemental Figure 3 provides sensitivity correction maps, and Supplemental Figure 4 patient-specific examples.

\section{DISCUSSION}

The 3-dimensional phase unwrapping method proved adequate for use in the UTILE-based 3-point Dixon decomposition in all investigated patients. The UTILE-based 3-point Dixon method was found comparable to mDixon and even better suited for MRI AC in the cerebrospinal fluid because of the
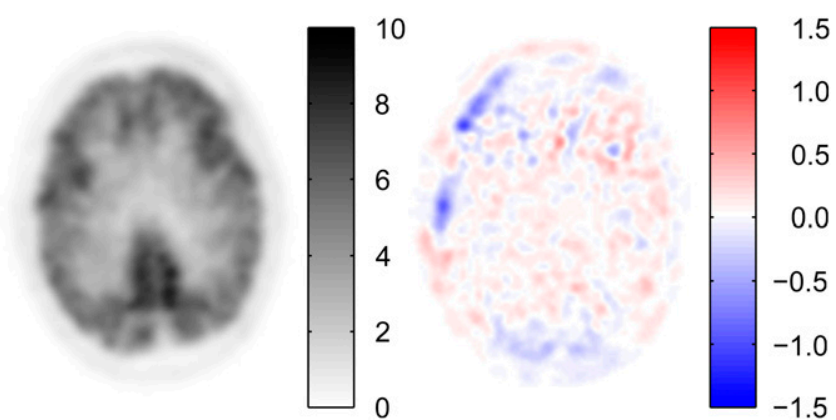

FIGURE 4. Corresponding transverse slices of PET UTILE (left) and difference image d UTILE (right) of patient P1 in SUV. Supplemental Figure 5 provides more examples.

lack of an inversion pulse. The difference image, especially in the areas of the orbits and the optical nerves, shows the good agreement of both methods.

More sophisticated water-fat separation techniques have been described, most notably the "iterative decomposition of water and fat with echo asymmetry and least-squares estimation" $(17,20)$. However, the application of this technique in this work is prevented mainly by 2 constraints: the difference in noise statistics between the UTE echo and the gradient echoes, and the echo times chosen to minimize overall acquisition durations.

Deriving an air mask from the UTE signal results in sufficient discrimination between air and other tissues. The discrimination of cortical bone produces good results but is challenging in areas of complex air-bone interfaces such as the paranasal sinuses (Fig. 2) or the vertebral column, supposedly because of susceptibility and partial-volume effects, combined with global thresholding of $\mathrm{d}_{\mathrm{UTE}}^{\mathrm{m}}$. Objects falsely classified as bone because of reduced relaxation times also include the tendon connecting the lower jaw bone with the temporal muscle.

The number of voxels in the tissue class intersections bone $^{\mathrm{CT}} \cap$ soft $^{\mathrm{UTILE}}$ and soft $^{\mathrm{CT}} \cap$ bone $^{\mathrm{UTILE}}$ highlights the necessity of bone segmentation improvements. These 2 bins represent, for example, areas of cranial bone and vertebral bodies falsely classified as soft tissue, and the voxels of the nasal sinuses incorrectly assigned to the bone class. In agreement, regions of maximum overestimation in the PET $\mathrm{UTILE}_{\mathrm{T}}$ dataset are found around the nasal sinuses and the pharynx (Supplemental Fig. 5, top row), where a too high attenuation coefficient yields an increase in the reconstructed activity.

Issues such as these, however, are not uncommon in the application of UTE signal acquisition for MRI-based AC and, especially, are not specific to the proposed combination of UTE acquisition and Dixon decomposition. In addition, acquisition times are only $30 \%$ longer than acquisition times of a single-echo UTE acquisition with otherwise identical parameters (164 s).

The choice of 4 segmentation classes and appropriate linear attenuation coefficients leads to a high correlation and good linearity between $\mathrm{s}_{\mathrm{UTILE}}$ and the reference $\mathrm{s}_{\mathrm{CT}}$, showing the advantage of 4-class tissue segmentation over 3-class 


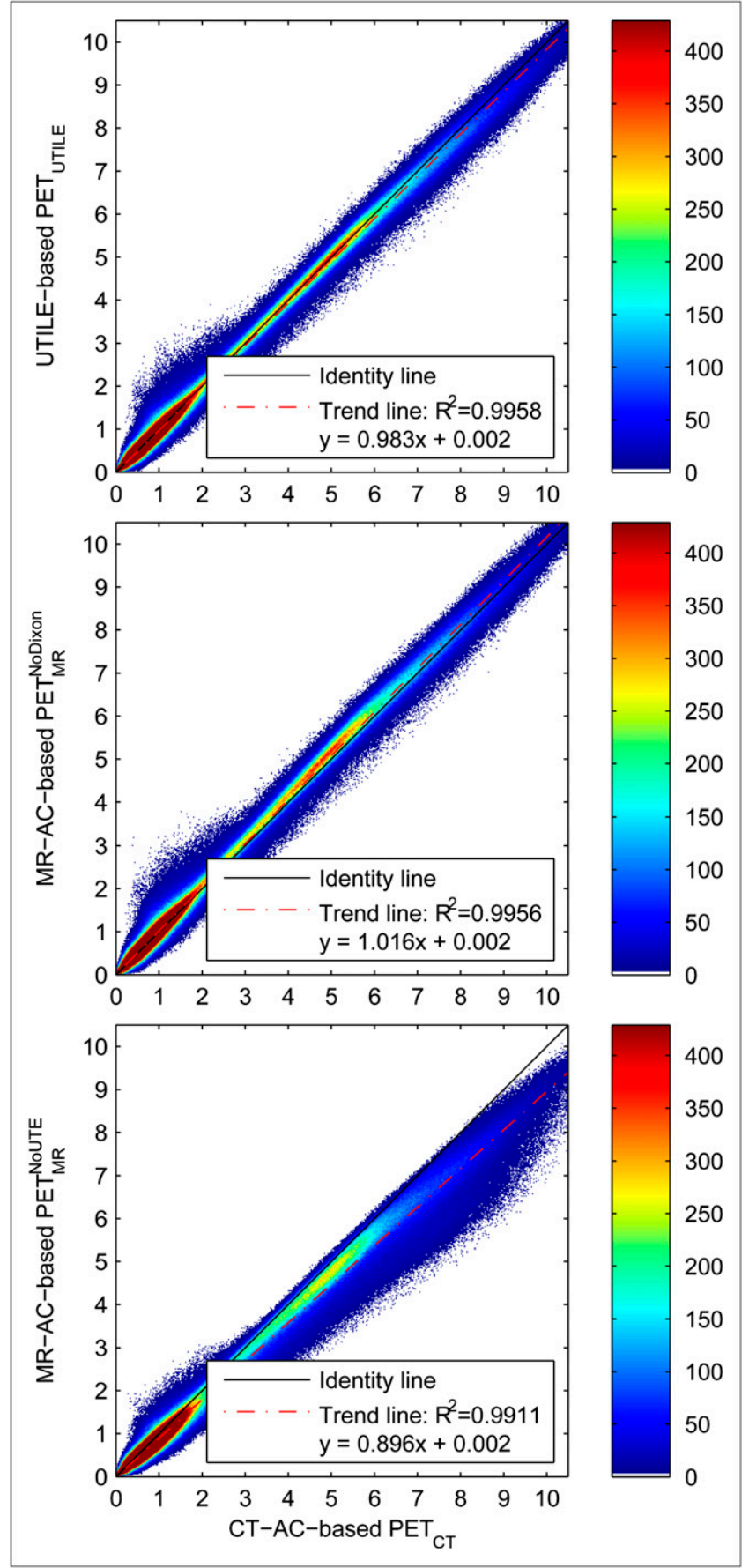

FIGURE 5. Joint histograms of PET UTILE (top), PET MRI NoDon (middle), and PET ${ }_{M R I}^{N o U T E}$ (bottom), all correlated with PET $\mathrm{CT}_{\mathrm{CT}}$ (aggregated over all patients). Supplemental Figure 6 provides patient-specific examples.

approaches. This translates into similar correlation and linearity findings in PET activities, although slight shifts in regression line slopes are to be observed. These shifts, however, do not have adverse effects on accuracies in brain region activities. As a result, the proposed method can be regarded as a potential alternative to CT AC, especially considering the possibilities of exploiting simultaneous PET/MRI acquisi-
TABLE 6

Coefficients of Determination $\left(R^{2}\right)$ Between PET ${ }_{\mathrm{CT}}$ and PET Using Different MRI-Based AC Methods*

\begin{tabular}{lccc}
\hline \multicolumn{1}{c}{ ID } & PET $_{\text {UTILE }}$ & PET $_{\text {MRI }}^{\text {NoDixon }}$ & PET $_{\text {MRI }}^{\text {NoUTE }}$ \\
\hline P1 & 0.9964 & 0.9962 & 0.9962 \\
P2 & 0.9954 & 0.9952 & 0.9907 \\
P3 & 0.9934 & 0.9931 & 0.9932 \\
P4 & 0.9973 & 0.9974 & 0.9931 \\
P5 & 0.9968 & 0.9966 & 0.9938 \\
P6 & 0.9970 & 0.9968 & 0.9909 \\
Aggregated & 0.9958 & 0.9956 & 0.9911 \\
\hline
\end{tabular}

*All correlations and differences between any $R^{2}$ in PETUTILE and corresponding value in any $\mathrm{PET}_{\mathrm{MRI}}$ are statistically significant $\left(P<10^{-6}\right)$.

tions for patient motion correction. Two MRI AC-specific particularities, however, will be briefly discussed:

Geometric distortions of the MRI data are not uncommon in the presence of metal or other field inhomogeneities. Although no localized distortions were detected in our study apart from metallic tooth implants, UTE acquisition parameters need to be carefully optimized such that the size of the imaged object is consistent across UTE and gradient echoes. On the used MRI system, the main parameters are trajectory delay and free induction decay image scaling samples. The former delays the start of the UTE sampling on the gradient ramp to compensate for eddy-current-induced gradient waveform delays; the latter influences the size of the image area reconstructed from the UTE signal. Although a calibration of these parameters is valid only for a specific set of sequence parameters, no related long-term drifts were observed in the experiments and a constant set of parameters was used throughout the study.

Furthermore, as can be seen from Figure 3 and Supplemental Figure 4, the choice of any predefined set of linear attenuation coefficients has to be seen as a compromise, as there are interpatient variations especially in bone attenuation coefficients. Some reasons for these interpatient variations include differences in bone mineral density, or the transition from water-fat fractions to mixed soft-tissue-adipose-tissue attenuation coefficients $\mu_{r}$. In this study, common values from the literature were used, rather than fitting attenuation coefficients to individual patients' CT data. Given a reliable segmentation, however, a PET reconstruction technique simultaneously estimating activity as well as attenuation (19) might have the potential of detecting inconsistencies in the attenuation-corrected PET activities and adapting the attenuation coefficients on a per-patient basis.

Finally, acknowledging CT AC as a reference technique, one should note that most comparisons were performed at CT resolution with a slice thickness of $5 \mathrm{~mm}$. It can be hypothesized that MRI AC performs more accurately than presented in this work if the isotropic MRI resolution is fully exploited. However, the current evaluation would not have benefitted from such improvement since the influences of 
TABLE 7

Regression Lines Between PET ${ }_{\mathrm{CT}}$ and PET Using Different MRI-Based AC Methods

\begin{tabular}{lccr}
\hline \multicolumn{1}{c}{ ID } & PET & PETILE $_{\text {MRI }}^{\text {NoDixon }}$ & PET NoUTE \\
\hline P1 & $1.019 x+0.001$ & $1.060 x+0.001$ & $0.962 x+0.000$ \\
P2 & $0.979 x+0.002$ & $1.009 x+0.003$ & $0.887 x+0.003$ \\
P3 & $1.000 x+0.000$ & $1.042 x+0.000$ & $0.913 x-0.001$ \\
P4 & $0.978 x+0.001$ & $1.011 x+0.001$ & $0.884 x+0.002$ \\
P5 & $1.005 x+0.000$ & $1.032 x+0.001$ & $0.938 x+0.001$ \\
P6 & $0.956 x+0.001$ & $0.989 x+0.001$ & $0.854 x+0.002$ \\
Aggregated & $0.983 x+0.002$ & $1.016 x+0.002$ & $0.896 x+0.002$ \\
\hline
\end{tabular}

specific MRI AC strengths (higher through-plane resolution, no metal streaking artifacts) or weaknesses (smaller field of view, bone misclassifications) are difficult to separate. Consequently, regions with metallic tooth implants were ignored in the evaluation, since deviations from the artifactimpacted CT AC results are to be expected but do not allow for assessment of MRI AC without gold standard activity measurements. Similarly, repeated interpolation of MRI data (from MRI to CT to PET resolution) was accepted to perform consistent comparisons.

Inclusion of $\mathrm{T}_{2}$ relaxation properties of tissue, currently ignored in the UTILE signal model, could enhance the accuracy of the water-fat decomposition. Also, calibration measurements of phantoms with known fractions of soft tissue to adipose tissue could improve the mapping of the relative water-fat fraction into the linear attenuation coefficient domain.

Several improvements in cortical bone segmentation are conceivable, including a more adequate computation of the phase offset from the complex nuclear magnetic resonance signal, a susceptibility artifact filter, and a nonglobal threshold segmentation of $\mathrm{d}_{\mathrm{UTE}}^{\mathrm{m}}$. Especially, the region of the paranasal sinuses and in particular the labyrinth of ethmoid require a higher robustness with regard to cortical bone segmentation. Additionally, rather than assigning a discrete linear attenuation coefficient to the entire segmentation class of cortical bone, it might be appropriate to account for different bone densities derived from the dynamics of the MRI signal decay.

To apply the proposed method to whole-body imaging, lung tissue as another important tissue class for whole-body AC has to be detected and an attenuation coefficient estimated.

\section{CONCLUSION}

This work demonstrates the feasibility of classificationbased MRI AC accounting for 4 different tissue classes-cortical bone, air, adipose tissue, and soft tissue — by combining the UTILE MRI sequence with dedicated postprocessing, exploiting the benefits of UTE acquisition as well as Dixon
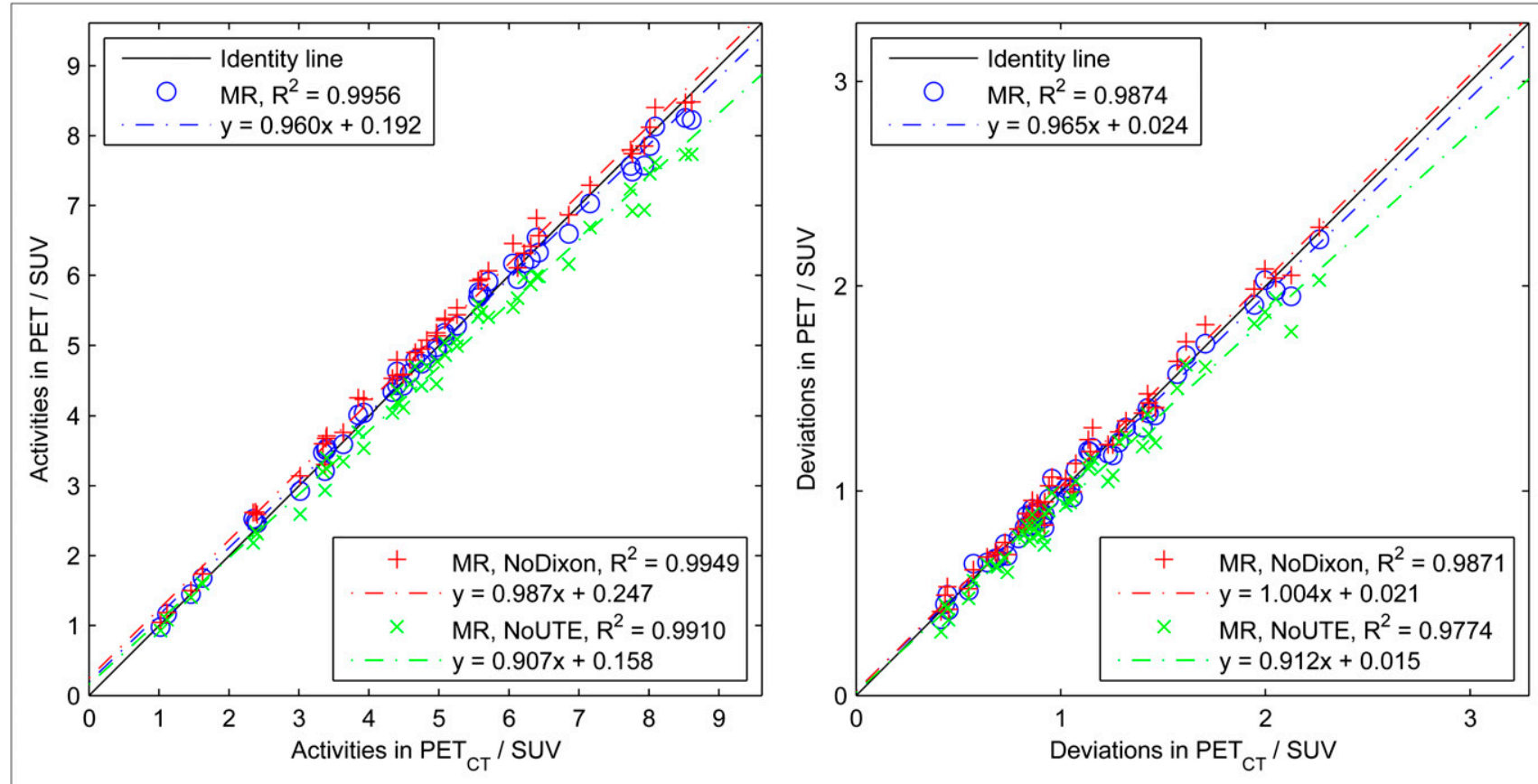

FIGURE 6. Scatterplot of regional mean values (left) and SD (right) of PET UTILE, PET $\mathrm{MRI}^{\mathrm{NoDixon}}$, and PET $\mathrm{MRI}^{\mathrm{NoUTE}}$, correlated with PET ${ }_{\mathrm{CT}}$ (all patients). 
decomposition. The good agreement with the reference CT AC method in patient head-and-neck examinations demonstrates the applicability of the proposed approach. Results were shown to be superior to 3-class postprocessing without UTE or Dixon components. The acquisition time of less than 4 min seems to be acceptable for a high-resolution AC, especially compared with the acquisition duration of single-echo UTE sequences at comparable resolution.

\section{DISCLOSURE STATEMENT}

The costs of publication of this article were defrayed in part by the payment of page charges. Therefore, and solely to indicate this fact, this article is hereby marked "advertisement" in accordance with 18 USC section 1734.

\section{ACKNOWLEDGMENT}

This research was supported by the European Community Seventh Framework Programme, project number 241711: "SUB nanosecond Leverage In PET/MR ImAging (SUBLIMA)." Volkmar Schulz is a full-time employee of Philips. No other potential conflict of interest relevant to this article was reported.

\section{REFERENCES}

1. Goerres GW, von Schulthess GK, Steinert HC. Why most PET of lung and headand-neck cancer will be PET/CT. J Nucl Med. 2004;45(suppl 1):66S-71S.

2. Schlemmer HP, Pichler BJ, Schmand M, et al. Simultaneous MR/PET imaging of the human brain: feasibility study. Radiology. 2008;248:1028-1035.

3. Judenhofer MS, Wehrl HF, Newport DF, et al. Simultaneous PET-MRI: a new approach for functional and morphological imaging. Nat Med. 2008;14:459-465.

4. Edelman RR, Hesselink J, Zlatkin M, Crues J, eds. Clinical magnetic resonance imaging. 3rd ed. Vol. 1. Physics, Instrumentation, and Advanced Techniques, Heart and Vascular System. New York, NY: Elsevier; 2006:288-319, 459-492.

5. Zaidi H, Montandon M, Slosman DO. Magnetic resonance imaging-guided attenuation and scatter corrections in three-dimensional brain positron emission tomography. Med Phys. 2003;30:937-948.
6. Hofmann M, Steinke F, Scheel V, et al. MRI-based attenuation correction for PET/MRI: a novel approach combining pattern recognition and atlas registration. J Nucl Med. 2008;49:1875-1883.

7. Schulz V, Torres-Espallardo I, Renisch S, et al. Automatic, three-segment, MRbased attenuation correction for whole-body PET/MR data. Eur J Nucl Med Mol Imaging. 2011;38:138-152.

8. Martinez-Möller A, Souvatzoglou M, Delso G, et al. Tissue classification as a potential approach for attenuation correction in whole-body PET/MRI: evaluation with PET/CT data. J Nucl Med. 2009;50:520-526.

9. Catana C, Van Der Kouwe A, Benner T, et al. Is accurate bone segmentation required for MR-based PET attenuation correction? [abstract]. Proc Intl Soc Mag Reson Med. 2009;17:593. Available at: http://cds.ismrm.org/protected/ 09MProceedings/files/00592.pdf. Accessed April 4, 2012.

10. Keereman V, Fierens Y, Broux T, et al. MRI-based attenuation correction for PET/MRI using ultrashort echo time sequences. J Nucl Med. 2010;51:812-818.

11. Robson MD, Gatehouse PD, Bydder M, Bydder GM. Magnetic resonance: an introduction to ultrashort TE (UTE) imaging. J Comput Assist Tomogr. 2003;27:825846.

12. Rahmer J, Börnert P, Groen J, Bos C. Three-dimensional radial ultrashort echotime imaging withT ${ }_{2}$ adapted sampling. Magn Reson Med. 2006;55:1075-1082.

13. Rahmer J, Blume U, Börnert P. Selective 3D ultrashort TE imaging: comparison of "dual-echo" acquisition and magnetization preparation for improving short-T2 contrast. MAGMA. 2007;20:83-92.

14. Dixon WT. Simple proton spectroscopic imaging. Radiology. 1984;153:189-194.

15. Glover GH, Schneider E. Three-point Dixon technique for true water/fat decomposition with $\mathrm{B}_{0}$ inhomogeneity correction. Magn Reson Med. 1991;18:371-383.

16. Cusack R, Papadakis N. New robust 3-D phase unwrapping algorithms: application to magnetic field mapping and undistorting echoplanar images. Neuroimage. 2002;16:754-764.

17. Reeder SB, Wen Z, Yu H, et al. Multicoil Dixon chemical species separation with an iterative least-squares estimation method. Magn Reson Med. 2004;51:35-45.

18. Kak AC, Slaney M. Algorithms for reconstruction with non-diffracting sources. In: Cotellessa R, ed. Principles of computerized tomographic imaging. New York, NY: IEEP Press; 1988:49-112.

19. Salomon A, Goedicke A, Schweizer B, Aach T, Schulz V. Simultaneous reconstruction of activity and attenuation for PET/MR. IEEE Trans Med Imaging. 2011;30:804-813.

20. Reeder SB, McKenzie CA, Pineda AR, et al. Water-fat separation with IDEAL gradient-echo imaging. J Magn Reson Imaging. 2007;25:644-652.

21. Beyer T, Kinahan PE, Townsend DW, Sashin D. The use of X-ray CT for attenuation correction of PET data. IEEE Nucl Sci Symp Conf Rec. 1994;4:15731577.

22. Burger C, Goerres G, Schoenes S, Buck A, Lonn AHR, von Schulthess GK. PET attenuation coefficients from CT images: experimental evaluation of the transformation of CT into PET 511-keV attenuation coefficients. Eur J Nucl Med Mol Imaging. 2002;29:922-927. 